\title{
CONHECIMENTO DE UMA EQUIPE DA ESTRATÉGIA SAÚDE DA FAMÍLIA SOBRE A POLÍTICA DE ATENÇÃO À SAÚDE MASCULINA
}

\author{
KNOWLEDGE OF A FAMILY HEALTH STRATEGY TEAM ON THE POLICY ON ATTENTION TO MEN'S HEALTH
}

\author{
CONOCIMIENTOS DE UN EQUIPO DE LA ESTRATEGIA SALUD FAMILIAR SOBRE LA POLÍTICA DE \\ ATENCIÓN A LA SALUD MASCULINA
}

\author{
Luís Paulo Souza e Souza ${ }^{1}$ \\ Eliane Rodrigues Almeida ${ }^{2}$ \\ Mariana Almeida Queiroz ${ }^{3}$ \\ José Rodrigo da Silva ${ }^{4}$ \\ Ana Augusta Maciel de Souza ${ }^{5}$ \\ Maria Fernanda Santos Figueiredo ${ }^{6}$
}

Resumo O estudo avalia o conhecimento de uma equipe da Estratégia Saúde da Família da cidade de Montes Claros, Minas Gerais, sobre a Política Nacional de Atenção Integral à Saúde do Homem. Trata-se de um estudo exploratório, descritivo, com abordagem qualitativa, caracterizando-se como estudo de caso, realizado com profissionais de uma unidade de saúde. Os dados foram coletados por meio de entrevista, no primeiro semestre de 2011. A maioria dos entrevistados apresentou conhecimento mínimo sobre a referida política, atribuído à limitação de informativos, inexistência de manuais do Ministério da Saúde e ausência de profissionais capacitados, ausência das esferas municipal, estadual ou federal. Parte dos entrevistados conhecia a composição da política, enquanto a totalidade sabia descrever o conhecimento real e amplo das características do processo saúde-doença masculino. Assim, torna-se importante considerar esse conhecimento prévio dos profissionais sobre essa população e investir em estratégias de capacitação deles, subsidiando assim a efetivação das ações de promoção, reabilitação e recuperação da saúde, diminuindo os indicadores de morbidade e mortalidade, como prevê a Política Nacional de Atenção Integral à Saúde do Homem.

Palavras-chave saúde do homem; atenção primária à saúde; conhecimento; serviços de saúde.
Abstract The study assesses the knowledge of a Family Health Strategy team from Montes Claros, state of Minas Gerais (Southeast Brazil), on the National Policy for Integral Attention to Men's Health. This is an exploratory, descriptive study with a qualitative approach, characterized as a case study conducted with professionals from a health care facility. The data were collected through interviews in the first half of 2011. Most respondents had minimal knowledge on this policy, which was attributed to the limitation in informational materials, the lack of guides from the Ministry of Health, of trained professionals, and to the absence of the municipal, state or federal spheres. Some of the respondents were aware of the composition of the policy, while all of them could describe actual and extensive knowledge of the characteristics of male health-disease process. Thus, it is important to consider these professionals' prior knowledge on this population and to invest in their training strategies, thereby subsidizing the effectiveness of health promotion, rehabilitation, and recovery actions, reducing morbidity and mortality, as prescribed by National Policy for Integral Attention to Men's Health. Keywords men's health; primary health care; knowledge; health services. 


\section{Introdução}

O campo da saúde pública admite que os homens apresentem riscos distintos para numerosos problemas de saúde. Esses riscos são demonstrados por meio de alguns indicadores epidemiológicos e comprovados por diversos pesquisadores (Laurenti, Jorge e Gotlieb, 2005).

Tratando-se de indicadores relacionados ao processo saúde-doença na população masculina, é possível destacar a mortalidade. Esta, por sua vez, pode ser delineada tanto pelos altos índices de morte prematura, principalmente em países em desenvolvimento como o Brasil, quanto por taxas de mortalidade nos homens com idades entre 15 e 44 anos, equivalendo duas vezes ou mais à mortalidade de mulheres desse mesmo grupo etário (Figueiredo, 2005).

Como possíveis causas para esse acometimento, apontam-se os fatores socioculturais referentes à construção da subjetividade masculina, bem como a injustiça distributiva em razão da ausência de equidade no atendimento das precisões de saúde. Tal quadro sugere uma provável assimetria nas políticas de saúde em relação ao homem, reincidindo em uma atenção precária nos serviços de saúde (Braz, 2005).

Diante dessas características, é plausível questionar a ausência de uma política para os homens, a qual poderia, a princípio, ser tida como uma questão simplesmente de gênero, pois, em geral, o cuidar de si e a valorização do corpo no sentido da saúde não foram questões apresentadas na socialização dos homens (Gomes, 2003).

É importante ressaltar que a reivindicação de uma política de saúde mais integral voltada para a população masculina poderia ser tomada como um movimento contrário ao empenho de promover programas de gênero destinados à saúde da mulher. Entretanto, a partir da própria dimensão de gênero se intercede uma abordagem também do masculino, porquanto tanto homens quanto mulheres necessitam ser vistos em sua singularidade e em sua diferença no âmbito das relações estabelecidas entre si (Gomes, 2003; Gomes et al., 2011a).

Conhecer as singularidades permite melhor aproximação do masculino e do feminino, conhecendo cada polo, não incluindo os homens apenas no debate sobre a saúde masculina, uma vez que, por consequência, conseguem-se ganhos para a saúde feminina em temas que só avançam tendo em vista a conquista da participação masculina em seu enfrentamento (Gomes, 2003; Schraiber, Gomes e Couto, 2005; Gomes et al., 201 la).

Reconhece-se que os agravos que acometem a população masculina constituem verdadeiros problemas de saúde pública, portanto necessitam de intervenções imediatas e eficazes. Nesse contexto, surge a Política Nacional de Atenção Integral à Saúde do Homem (PNAISH), uma iniciativa do Ministério da Saúde, alinhada à Política Nacional de Atenção Básica 
(PNAB) e que traz consigo estratégias que buscam minimizar a resistência masculina a respeito dos cuidados com a saúde (Brasil, 2009).

Nesse sentido, com base no quadro atual da saúde nacional, pode-se destacar também o recente desenvolvimento da PNAISH - lançado em primeira versão em agosto de 2008 pelo Departamento de Ações Programáticas e Estratégicas, o qual se subordina à Área Técnica de Saúde do Homem -, bem como o seu caráter fundamental, porque tal política de saúde não se limita a uma questão simplesmente de gênero, mas, ao contrário, torna-se medida aplicável (Carrara, Russo e Faro, 2009). Esses autores informam que as estratégias da PNAISH trabalham a humanização e a qualidade na assistência de saúde dos homens, fundamentando-se preferencialmente em ações educativas propiciadoras da libertação de seus próprios preconceitos e incentivo ao autocuidado.

Assim, além de embasamento teórico e conhecimento específico, é preciso profissionais da saúde com habilidades técnicas, desenvolvendo uma abordagem holística para assegurar um cuidado resolutivo e seguro (Albano, Basílio e Neves, 2010). Desse modo, justifica-se verificar o conhecimento de profissionais acerca da PNAISH, pois tal conhecimento possibilita à equipe um trabalho efetivo na prevenção de doenças ou agravos que mais incidem nos homens e o desenvolvimento de estratégias de promoção da saúde, minimizando indicadores de morbidade e mortalidade masculina.

Somando esses fatores e considerando suas respectivas lacunas, identifica-se a necessidade de verificar o conhecimento de profissionais da atenção primária, a 'porta de entrada' do serviço de saúde, sobre a PNAISH no que concerne a diretrizes, direcionamento e especificidades das ações; reconhecimento das peculiaridades da população masculina; bem como à estrutura organizacional da atenção primária, preferencialmente quanto à promoção da saúde e prevenção de agravos ou doenças para subsidiar o desenvolvimento efetivo dessa política. A problemática levantada pelos pesquisadores foi: qual o conhecimento apresentado por profissionais da Estratégia Saúde da Família (ESF) sobre a PNAISH?

Assim, com base no exposto, o presente estudo objetivou verificar o conhecimento apresentado pela equipe da ESF de um bairro da cidade de Montes Claros, situada ao norte do estado de Minas Gerais, acerca da PNAISH.

\section{Metodologia}

Realizou-se um estudo exploratório, descritivo, com abordagem qualitativa, caracterizando-se como estudo de caso, pois focalizou-se em profissionais de apenas uma unidade de saúde da família. Alves-Mazzotti (2006) esclarece 
que estudos de caso são usados como etapas exploratórias na pesquisa de fenômenos pouco investigados, os quais exigem estudo aprofundado de um número reduzido de casos, que leve à identificação de categorias de observação ou à geração de hipóteses para estudos posteriores, como é o caso da temática saúde do homem na ESF.

A unidade de saúde da família escolhida situa-se na cidade de Montes Claros, norte do estado de Minas Gerais, Brasil, e na ocasião era composta por um médico, um enfermeiro, um técnico de enfermagem e cinco agentes comunitários de saúde.

Os profissionais foram selecionados mediante os seguintes critérios de inclusão: ser profissional de saúde, atuar na ESF pesquisada há pelo menos seis meses e aceitar participar da pesquisa. Anteriormente à coleta dos dados, realizou-se um estudo-piloto com profissionais atuantes em outra unidade de atenção primária à saúde (UAPS) de Montes Claros, visando verificar a adequação do roteiro da entrevista no que diz respeito à linguagem adequada, coerente ao entendimento e formulação de indagações pertinentes ao alcance dos objetivos propostos.

A coleta de dados ocorreu no primeiro semestre de 2011, sendo realizada por um dos autores, no local de trabalho dos profissionais de saúde, após a leitura dos objetivos da pesquisa e assinatura do termo de consentimento livre e esclarecido, por meio de entrevista, com as seguintes questões norteadoras: "Fale-me sobre a Política Nacional de Atenção Integral à Saúde do Homem"; "Conte-me sobre os princípios que orientam a Política Nacional de Atenção Integral à Saúde do Homem"; "Diante das elevadas taxas de morbidade e mortalidade masculina, fale pra mim sobre as ações principais que a Política Nacional de Atenção Integral à Saúde do Homem objetiva trabalhar"; "Conte para mim sobre os temas fundamentais a serem trabalhados com a população masculina"; "Fale pra mim sobre os fatores favoráveis e não favoráveis à implantação desta política".

As entrevistas foram gravadas e, em seguida, transcritas, o que possibilitou o registro fidedigno de todas as informações fornecidas pelos entrevistados. Os participantes foram identificados com legendas, mantendo o anonimato.

Para análise dos dados, foi utilizada a técnica de análise do conteúdo tipo categorial (Bardin, 2009), a qual apresenta os seguintes passos: pré-análise - organização do corpus de conhecimento para análise; leitura flutuante; leitura exaustiva; escolha e codificação das unidades de análise; elaboração das categorias, que foram: "Relacionando o conhecimento dos profissionais com o processo saúde e doença do homem" e "Relacionando o conhecimento dos profissionais à Política Nacional de Atenção Integral à Saúde do Homem", buscando-se as relações entre elas. 
Ressalte-se que esta pesquisa foi aprovada pelo Comitê de Ética em Pesquisa da Universidade Estadual de Montes Claros (Unimontes), por meio do parecer número 2229/2011.

\section{Resultados e discussão}

Participaram do estudo sete profissionais de saúde que integravam a unidade escolhida (um médico, um enfermeiro e cinco agentes comunitários de saúde). Os discursos dos entrevistados revelaram duas categorias empíricas, as quais serão discutidas a seguir.

\section{Relacionando o conhecimento dos profissionais com o processo saúde e doença do homem}

Com base nas respostas, nota-se que alguns profissionais denotaram pouca intimidade com a composição da PNAISH, justificada inclusive pela ausência de difusão das diretrizes que a compõem. Contudo, todos conceituaram e exemplificaram situações e características peculiares da saúde masculina obtidas pelas próprias vivências e observações.

\footnotetext{
Não sei, não, não sei nem do que se trata (E2).

É uma novidade para o PSF, já vi muito assim em televisão, né? A propaganda falando da saúde do homem (E3).

O homem não procura o serviço, adoece e só vem quando tá mal (E1).
}

Esse conhecimento prévio dos fatores, associado a atitudes ou mesmo omissões de saúde e doença dos homens e do próprio serviço de atenção primária, favoreceu a criação de subcategorias que constituem considerações importantes a serem trabalhadas na implantação e aplicabilidade da PNAISH.

Os homens, na construção de gênero, expõem-se a riscos que acabam por intervir em sua saúde (Figueiredo, 2005). Muitas vezes, eles apresentam comportamentos que não são saudáveis, quando relacionados a uma masculinidade idealizada proveniente da cultura masculina. Tal fato é reconhecido pelos entrevistados, como observado a seguir:

É interessante, porque homem geralmente é difícil de sair nas unidades procurando. Quando procura, é porque a situação já está muito relevante (E1). 
O acometimento pelas condições crônicas se dá, na maioria, nas mulheres, porém quando é levada em conta a gravidade da doença, a população masculina tende a ter maiores consequências como as doenças crônicas fatais (Figueiredo, 2005). É reconhecido que os homens ingressam no sistema de saúde por meio da atenção terciária, o que acaba por trazer o agravo da sua condição pelo atraso do cuidado. Logo, é necessário fortificar e também qualificar a atenção primária para garantir a promoção da saúde e a prevenção de doenças que, se forem detectadas em seu início, têm elevada sua chance de cura (Brasil, 2009), o que também é percebido pelos entrevistados:

(...) porque o homem geralmente ele mesmo esquece de si, ele não cuida da própria saúde (E2).

Além da questão cultural masculina que persiste nos tempos atuais, tem-se a questão das políticas públicas voltadas, em sua maioria, para as mulheres e crianças, sendo este um fator que dificulta o acesso dos homens ao serviço de saúde da atenção primária (Couto et al., 2010). Tal fato também foi considerado pelos profissionais de saúde:

As políticas públicas só trabalham com as mulheres, as crianças. E esquecem que também existe o homem, e sem saúde não tem como o homem trabalhar, manter a sua família. O homem, sem a sua saúde, ele que é o provedor, vai desestruturar toda a sua família, então ele é a força, a base de toda a sua família (E3).

Segundo o relato anterior, um argumento justificável à PNAISH é a condição de provedor assumida prioritariamente pelos homens no seu grupo familiar. Destarte, um processo de adoecimento masculino compromete psicossocial e economicamente o homem e toda a sua família, evidenciando que a criação e a implantação da PNAISH devem contemplar o homem em todos os seus aspectos, fazendo compreender a necessidade de ele próprio enxergar a importância da sua saúde e com isso mudar o paradigma segundo o qual, estando doente, ele se apresentará como um ser fraco diante da condição na qual se encontrar (Figueiredo e Schraiber, 2011).

O cuidado com a saúde não é valorizado como uma questão importante nas vivências masculinas; assim, os homens têm dificuldades em expressar suas necessidades de saúde, procurando menos do que as mulheres os serviços de saúde (Figueiredo e Schraiber, 2011). Existem várias suposições ou até mesmo justificativas para explicar a ausência dos homens nos serviços de atenção primária, como a preferência deles por serviços que atendam mais rapidamente às suas necessidades, como as farmácias e os prontos-socorros (Brasil, 2009).

Nesses estabelecimentos, os homens conseguem expor os seus problemas com uma facilidade maior, sendo que consideram a unidade de atenção 
primária à saúde como um espaço feminilizado, constituído de profissionais, em sua maioria, representados por mulheres e frequentado também por pessoas desse sexo. Assim, a população masculina se sentiria como não pertencendo àquele espaço. Tais fatores ganham força quando associados à falta de políticas públicas para a população masculina (Brasil, 2009).

Outra explicação para a não busca do homem pelo serviço básico de saúde, segundo Fontes e colaboradores (2011), deve-se ao fato de o horário de funcionamento do serviço coincidir com a sua carga horária de trabalho. Porém, os horários do funcionamento das instituições públicas nem sempre são conciliáveis com os horários das pessoas que se encontram no mercado formal de trabalho, conforme também verificado:

Como o homem é trabalhador e a unidade fica aberta em horário comercial, ele não procura o serviço, que é o horário que ele não pode, e à noite ele não pode porque está estudando, e aí que horas ele vai vir, se ele já não tem o hábito? Se você [profissional] não tem o hábito de trabalhar, o homem ele também não tem o hábito de trabalhar as ações (E4).

Até certa idade, os idosos procuram mais a unidade, a gente tem mais facilidade em trabalhar com eles, eles são mais conscientes, mas até os 40 anos os homens são mais preocupados com o trabalho (E5).

Com base nessa observação, indaga-se: como as mulheres, que estão alcançando rapidamente um lugar de destaque na sociedade com a conquista do mercado de trabalho, conseguem ter o tempo necessário para cuidar de sua saúde?

Laurenti, Jorge e Gotlieb (2005) informam que a mulher, em contraposição ao homem, tem sido alvo de numerosos estudos e políticas. Entretanto, quando analisados dados de morbidade e mortalidade, aponta-se a necessidade de incluir os homens em políticas que contemplem a sua singularidade. A mulher adoece em número maior, porém como a questão do cuidar da saúde vem de hábitos e culturas, ela se preocupa, em mais proporção, com a sua saúde - o que é explicado por ela ser preparada, desde a infância, para cuidar de si mesma, para que, posteriormente, possa cuidar de seus filhos e esposo. Notam-se tais questões na fala a seguir:

Como o homem não tem o hábito de prevenir, nem de procurar o serviço, nem a mãe trabalha com ele a necessidade, então o homem não vem (E6).

Essa ausência masculina na atenção primária pode estar ligada à prevenção, um costume não adotado pela população masculina. No entanto, para as mulheres, tal ação é um cuidado, o qual ultrapassa gerações (Laurenti, Jorge e Gotlieb, 2005). Outra barreira social que dificulta o acesso dos homens 
aos serviços de saúde pode ser explicada pelo modelo de masculinidade idealizado como invulnerabilidade e comportamentos de risco, oriundos da cultura masculina (Figueiredo, 2005).

A organização e a rotina dos serviços influenciam fortemente na reprodução do imaginário social de gênero. Tal fato, por sua vez, repercute na atenção oferecida à população (Couto et al., 2010).

Desse modo, Machado e colaboradores (2007) propõem que as ações profissionais na atenção à saúde masculina devem abordar estratégias de prevenção de doenças e agravos, reabilitação e manutenção da saúde relacionados às principais causas de morbidade e mortalidade que acometem essa população - mas também estratégias de aprendizagem favoráveis ao diálogo, à troca, à transdisciplinaridade entre diferentes saberes formais ou informais, que contribuam para as ações de promoção da saúde individual e coletiva. Este fator também foi considerado no seguinte depoimento:

Você precisa trabalhar principalmente os hábitos saudáveis, atividade física, prevenção, nos homens acima de 40 anos a questão do câncer de próstata. Os homens morrem por doenças cardiovasculares, causas externas como os acidentes, mortes violentas, e que a gente deveria trabalhar com esse homem a própria sensibilização de hábitos saudáveis, principalmente a questão da alimentação, hábitos de vida, melhor bem-estar, condições de vida, e isso mudaria o paradigma de visão do homem (E4).

O processo de educação da população masculina deve se iniciar na infância, uma vez que favorece tanto a construção gradual e crescente da prevenção de agravos e doenças - como as cardiovasculares, que requerem manutenção de atividade física regular, controle dietético durante toda a vida - quanto a abordagem pró-ativa sobre os fatores de risco que podem comprometer a saúde do indivíduo, bem como seu grupo familiar (Junior e Lima, 2009). Na fala a seguir se observam tais afirmações:

(...) então se você trabalha desde a infância hábitos saudáveis, você previne o câncer, a obesidade, alterações cardíacas, e se vier a ter alguma alteração você pode evitar para que não complique (E4).

Nesse contexto, o Ministério da Saúde (Brasil, 2009) explica que um dos objetivos da PNAISH refere-se à captura precoce da população masculina nas atividades de prevenção primária a doenças cardiovasculares e cânceres, entre outras patologias recorrentes.

Assim, considerar o processo saúde e doença dos homens pela ótica dos fatores culturais, sociais e ideológicos relacionados à educação dessa população desde a infância torna-se fundamental, porquanto assim é possível 
compreender e garantir o acompanhamento das múltiplas causas que envolvem o adoecer, identificar ações prioritárias e formar conceitos de autocuidado responsáveis em todas as fases da vida (adolescência, juventude, fase adulta e velhice) (Junior e Lima, 2009). A educação em saúde também promove a difusão do cuidado a todo o grupo familiar, fornecendo, portanto, subsídios não apenas ao homem, mas a toda família, a fim de aderir a terapêuticas, superar agravos e controlar fatores de risco específicos da dinâmica familiar (Gomes et al., 2011b).

\section{Relacionando o conhecimento dos profissionais à PNAISH}

Quando indagados sobre princípios, ações, vivências e processo de implantação da PNAISH, alguns profissionais não expressaram nenhum conhecimento. Outros, por sua vez, apresentaram conhecimento limitado e conhecimento da essência da política, o que propiciou afirmações e associações dos principais aspectos dela.

A ausência de indivíduos do sexo masculino na UAPS não deve ser considerada como uma falta de responsabilidade somente dos homens ou apenas dos serviços de saúde da atenção primária, mas sim compreendida como problema que envolve aspectos particulares de cada um, tais como: os homens como sujeitos desafiados com as diferentes dimensões da vida; a organização dos serviços de saúde para atender os seus usuários diante de suas particularidades; e a ligação estabelecida entre os homens e os serviços (Figueiredo, 2005). Esses elementos foram percebidos no seguinte discurso:

A política nacional de atenção integral à saúde do homem propõe moldar a visão tanto dos profissionais da saúde em abordar o homem quanto dos próprios homens em aprender a prevenir (E4).

A corresponsabilização do cuidado entre profissional e população-alvo torna-se um processo de trabalho novo e promissor, pois possibilita a construção social de demandas e necessidades em saúde. Tais fatores resultam em reorganização das práticas visando à superação de ações meramente assistenciais e no fortalecimento das ações de promoção em saúde (Couto et al., 2010) - o que pode ser observado na fala a seguir:

(...) então é interessante esse trabalho em relação aos homens porque eles têm que se conscientizar que eles também, assim como as mulheres, precisam também de cuidados (E1).

É um desafio incluir a participação dos homens nas ações de saúde por inúmeros motivos, como por exemplo o cuidar de si e a valorização do corpo, 
questões que, infelizmente, de forma habitual não são apresentadas no processo de criação dos homens (Schraiber, Gomes e Couto, 2005).

A articulação da PNAISH à atenção primária é sustentável e coerente, pois as unidades de atenção primária à saúde constituem um modelo assistencial que permite o acompanhamento contínuo do cliente (visão integrada baseada na pessoa, ênfase em prevenção de doenças e trabalho multiprofissional), a educação e o incentivo quanto à necessidade de adotar hábitos preventivos, ações em que se fundamenta a iniciativa de atenção integral à saúde masculina (Junior e Lima, 2009).

Além disso, a integração da PNAISH à atenção primária justifica-se, pois se trata de uma abordagem de base que determina o trabalho de todos os outros níveis de atenção dos sistemas de saúde (Carrara, Russo e Faro, 2009). Notam-se tais conhecimentos por parte do profissional na fala a seguir:

Na realidade, a saúde do homem está contemplada na atenção primária, a política nacional é a atenção integral tanto na questão emocional, física (E7).

O relato anterior se valida em Brasil (2009), documento que acrescenta a proposição da PNAISH de qualificar a saúde da população masculina na perspectiva de linhas de cuidado que resguardem a integralidade da atenção. Ou seja, essa integralidade é possível mediante a abordagem do homem como sujeito do processo saúde-doença e consideração das diferenças de idade, condição socioeconômica, étnico-racial, orientação sexual e identidade de gênero, para então direcionar todas as ações da atenção primária - promoção e manutenção da saúde, prevenção de doenças e agravos, reabilitação e recuperação - baseadas em especificidade, parcerias na comunidade, família e intersetorialidade (Couto et al., 2010).

Dentre os princípios e diretrizes estipulados pela PNAISH, desde a sua criação em 2008 (Brasil, 2009), destacam-se a implantação e a implementação de protocolos clínicos e terapêuticos em consonância com as diretrizes nacionais e estaduais; qualificação das equipes de saúde para execução das ações propostas na política; e inclusão de temas ligados à atenção integral à saúde do homem na educação permanente dos trabalhadores do Sistema Único de Saúde. Tais ações, por sua vez, mesmo em 2011, ano da pesquisa, não foram concretizadas, como se observa nos relatos a seguir:

Mas ela, até então, nas nossas unidades, está mais na teoria do que na prática, mas porque a política ainda não trouxe nenhuma forma de diretrizes e nenhuma maior sensibilização de como nós vamos trazer esse homem para a unidade e trabalhar a questão da prevenção. Então a gente continua a fazer da mesma forma, sendo que existe uma política nova, mas que o Ministério nunca capacitou as unidades (E4). 
Na televisão passa, como já falei. Aqui na unidade é novidade (E3).

O atraso na qualificação profissional para atender às questões de saúde e doença da população masculina compromete o desenvolvimento do processo de trabalho da equipe multiprofissional no âmbito da realização prática dos atendimentos aos homens; a divulgação efetiva de informações que difundam a PNAISH; e a aplicação da humanização e diminuição dos índices de morbidade e mortalidade masculinos, princípios e objetivos inerentes à PNAISH.

Em pesquisa realizada pelo Ministério da Saúde, observou-se que nenhum dos entrevistados apresentava conhecimento preciso sobre a PNAISH. Contudo, eles enfatizavam a necessidade de associação da capacitação profissional à disponibilização de infraestrutura (recursos materiais e pessoais, além de condições de ambiente físico satisfatório) adequada à assistência qualitativa (Brasil, 2009).

Assim, para habilitar o profissional, faz-se necessário vincular mudanças de ordem gerencial, organizacional e estrutural (Gomes et al., 2011b), enfatizando a participação de mais setores da sociedade, e assim articular tecnologia, acolhimento, melhor comunicação e condições de trabalho. Corroborando tal raciocínio, Aguiar e Almeida (2012) enfatizam que é preciso mobilização dos órgãos gestores da saúde para implementação de ações de divulgação da PNAISH. E, mais importante, capacitação dos profissionais para atender os homens alcançados por essa divulgação, para que a política não seja desacreditada pela população masculina ao encontrar profissionais despreparados para atendê-la em suas necessidades.

\section{Considerações finais}

Verificou-se que parte dos profissionais apresentou conhecimento insatisfatório ou mesmo nenhum conhecimento a respeito da PNAISH. Essa constatação pode ser explicada segundo os próprios profissionais ao relatarem a ausência de capacitação das esferas do governo, seja municipal, seja estadual ou federal, tanto no fornecimento de materiais didáticos e publicações (manuais) quanto na informação direta de profissionais habilitados. Conforme o plano de ação nacional, essa política já deveria estar mais próxima da realidade das UAPS, no que diz respeito à elaboração de planos de ações municipais, reorganização e planejamento do processo de trabalho da equipe multiprofissional, bem como sua qualificação.

Ficou evidenciado que mesmo não conhecendo a composição da política, a equipe conseguia identificar por meio das vivências e dinâmica do serviço as ações e necessidades que permeiam o processo saúde e doença dos homens. 
Tal fato sugere a importância de se considerar esse conhecimento prévio dos profissionais sobre a população masculina, investindo em estratégias de capacitação, a fim de que haja implantação adequada e efetiva da política, relacionada à organização estrutural e qualificação profissional.

É coerente afirmar que mesmo com tantos obstáculos, como barreiras institucionais e sociais a serem superadas, acredita-se na possibilidade, com união e empenho veemente dos órgãos responsáveis, de obter resultados favoráveis, como a redução da morbidade e da mortalidade masculina, pois a presente política traz premissas essenciais, como intersetorialidade, humanização, mudança de paradigma e educação em saúde, para uma atenção integral e de qualidade da população masculina.

\section{Colaboradores}

Eliane Rodrigues Almeida e Mariana Almeida Queiroz participaram da concepção, coleta de dados, análise de dados e redação do artigo. Luís Paulo Souza e Souza e Ana Augusta Maciel de Souza participaram da concepção, análise de dados e redação do artigo. José Rodrigo da Silva e Maria Fernanda Santos Figueiredo atuaram na análise de dados, redação do artigo e revisão final. Declaramos que não há conflito de interesses na elaboração do trabalho.

Resumen El estudio evalúa los conocimientos de un equipo de la Estrategia Salud Familiar de Montes Claros, Minas Gerais (Sureste de Brasil), sobre la Política Nacional de Atención Integral a la Salud del Hombre. Se trata de un estudio exploratorio, descriptivo, con enfoque cualitativo, que se caracteriza como un estudio de caso, realizado con profesionales de una unidad de salud. Los datos fueron recolectados a través de entrevistas, en el primer semestre de 2011. La mayoría de los entrevistados presentaban un conocimiento mínimo sobre la referida política, atribuido a la escasez de informativos, inexistencia de manuales del Ministerio de Salud y falta de profesionales capacitados, ausencia de las esferas municipal, estatal o federal. Parte de los entrevistados conocían la composición de la política, mientras que la totalidad sabía describir el conocimiento real y amplio de las características del proceso salud-enfermedad masculino. Por lo tanto, es importante tener en cuenta estos conocimientos previos de los profesionales sobre esta población e invertir en estrategias de capacitación de éstos, para materializar las acciones de promoción, rehabilitación y recuperación de la salud, disminuyendo los indicadores de morbilidad y mortalidad, como lo prevé la Política Nacional de Atención Integral a la Salud del Hombre.

Palabras clave salud del hombre; atención primaria de la salud; conocimiento; servicios de salud. 


\section{Notas}

1 Universidade Federal de Minas Gerais, Belo Horizonte, Minas Gerais, Brasil.

Mestrando pela Escola de Enfermagem da Universidade Federal de Minas Gerais.

$<$ luis.pauloss@hotmail.com>

Correspondência: Rua Doze, 47, Santo Antônio, CEP 39402-285, Montes Claros, Minas Gerais, Brasil.

2 Faculdades Integradas Pitágoras, Montes Claros, Minas Gerais, Brasil. Graduada em Enfermagem por Faculdades Integradas Pitágoras.

<elianealmeida18@yahoo.com.br>

3 Faculdades Integradas Pitágoras, Montes Claros, Minas Gerais, Brasil.

Graduada em Enfermagem por Faculdades Integradas Pitágoras.

<mayalq@yahoo.com.br>

4 Universidade Federal de Minas Gerais, Belo Horizonte, Minas Gerais, Brasil.

Mestrando em Promoção de Saúde e Prevenção da Violência na Universidade Federal de Minas Gerais. <rodrigomaiss@yahoo.com.br>

5 Universidade Estadual de Montes Claros, Montes Claros, Minas Gerais, Brasil.

Mestre em Saúde Coletiva pela Universidade Federal de São Paulo.

<anamaciel@uai.com.br>

6 Universidade Estadual de Montes Claros, Montes Claros, Minas Gerais, Brasil.

Doutoranda em Ciências da Saúde na Universidade Estadual de Montes Claros. $<$ nanda_sanfig@yahoo.com.br>

\section{Referências}

AGUIAR, Milena C.; ALMEIDA, Obertal S. A implantação da Política Nacional de Atenção Integral à Saúde do Homem no Brasil: um desafio para a saúde pública. Diálogos \& Ciência, Feira de Santana, v. 10, n. 31, p. 144-147, 2012.

ALBANO, Bruno R.; BASÍlio, Márcio C.; NEVES, Jussara B. Desafios para a inclusão dos homens nos serviços de atenção primária à saúde. Revista Enfermagem Integrada, Ipatinga, v. 3, n. 2, p. 554-563, 2010.

ALVES-MAZZOTTI, Alda J. Usos e abusos dos estudos de caso. Cadernos de Pesquisa, São Paulo, v. 36, n. 129, p. 637-651, 2006.
BARDIN, Laurence. Análise de conteúdo. Lisboa: Edições 70, 2009.

BRASIL. Ministério da Saúde. Política Nacional de Atenção Integral à Saúde do Homem: princípios e diretrizes. Brasília: Ministério da Saúde, 2008. Disponível em: <http:// bvsms.saude.gov.br/bvs/publicacoes/politica_ nacional_atencao_homem.pdf > . Acesso em: 21 mar. 2010

BRAZ, Marlene. A construção da subjetividade masculina e seu impacto sobre a saúde do homem: reflexão bioética sobre justiça distributiva. Ciência \& Saúde Coletiva, Rio de Janeiro, v. 10, n. 1, p. 97-104, 2005. 
CARRARA, Sérgio; RUSSO, Jane A.; FARO, Livi. A política de atenção à saúde do homem no Brasil: os paradoxos da medicalização do corpo masculino. Physis: Revista de Saúde Coletiva, Rio de Janeiro, v. 19, n. 3, p. 659-678, 2009.

COUTO, Márcia T. et al. O homem na atenção primária à saúde: discutindo (in)visibilidade a partir da perspectiva de gênero. Interface: Comunicação, Saúde, Educação, Botucatu, v. 14, n. 33, p. 257-270, 2010.

FIGUEIREDO, Wagner S.; SCHRAIBER, Lilia B. Concepções de gênero de homens usuários e profissionais de saúde de serviços de atenção primária e os possíveis impactos na saúde da população masculina, São Paulo, Brasil. Ciência \& Saúde Coletiva, Rio de Janeiro, v. 16, n. 1, p. 935-944, 2011.

FIGUEIREDO, Wagner. Assistência à saúde dos homens: um desafio para os serviços de atenção primária. Ciência \& Saúde Coletiva, Rio de Janeiro, v. 10, n. 1, p. 105-109, 2005.

FONTES, Wilma D. et al. Atenção à saúde do homem: interlocução entre ensino e serviço. Acta Paulista de Enfermagem, São Paulo, v. 24, n. 3, p. 430-433, 2011.

GOMES, Romeu. Sexualidade masculina e saúde do homem: proposta para uma discussão. Ciência \& Saúde Coletiva, Rio de Janeiro, v. 8, n. 3, p. 825-829, 2003.
GOMES, Romeu et al. A atenção básica à saúde do homem sob a ótica do usuário: um estudo qualitativo em três serviços do Rio de Janeiro. Ciência \& Saúde Coletiva, Rio de Janeiro, v. 16, n. 11, p. 4.513-4.521, 2011 b.

GOMES, Romeu et al. Os homens não vêm! Interpretação dos profissionais de saúde sobre ausência e ou invisibilidade masculina nos serviços de atenção primária do Rio de Janeiro. Ciência \& Saúde Coletiva, Rio de Janeiro, v. 16, supl. 1, p. 983-992, 201 la.

JUNIOR, Eduardo A.; LIMA, Hermínio S. Promoção da saúde masculina na atenção básica. Pesquisa em Foco, Maranhão, v. 17, n. 2, p. 32-41, 2009.

LAURENTI, Ruy; JORGE, Maria H. P. M.; GOTLIEB, Sabina L. D. Perfil epidemiológico da morbimortalidade masculina. Ciência \& Saúde Coletiva, Rio de Janeiro, v. 10, n. 1, p. 35-46, 2005.

MACHADO, Maria F. A. S. et al. Integralidade, formação de saúde, educação em saúde e as propostas do SUS: uma revisão conceitual. Ciência \& Saúde Coletiva, Rio de Janeiro, v. 12, n. 2, p. 335-342, 2007.

SCHRAIBER, Lilia B.; GOMES, Romeu; COUTO, Márcia T. Homens e saúde na pauta da saúde coletiva. Ciência \& Saúde Coletiva, Rio de Janeiro, v. 10, n. 1, p. 7-11, 2005.

Recebido em 20/03/2012

Aprovado em 16/09/2013 